\title{
Manifestação Clínica, Modelos de Classificação e Fatores de Risco/Proteção para Psicopatologias na Infância e Adolescência
}

\author{
Fabiana Vieira Gauy \\ Universidade de Brasília, Brasília, Distrito Federal, Brasil \\ Marina Monzani da Rocha ${ }^{1}$ \\ Instituto de Ciências Humanas da Universidade Paulista, São Paulo, São Paulo, Brasil
}

\section{Resumo}

Ainda que a atenção à saúde mental infanto-juvenil tenha aumentado desde o início do século XXI, os trabalhos nessa área ainda são incipientes e há poucas publicações na literatura sobre intervenção em saúde mental voltadas a essa faixa etária em comparação com os trabalhos realizados com o público adulto. Questões relativas à psicopatologia infanto-juvenil possuem singularidades e merecem considerações distintas quanto à evolução, à classificação e ao tratamento, levando-se sempre em consideração o estágio de desenvolvimento individual. Este artigo tem como objetivo abordar três aspectos associados à saúde mental infanto-juvenil e de grande impacto tanto no diagnóstico como no prognóstico de dificuldades emocionais e comportamentais, a saber: (a) heterogeneidade das manifestações clínicas; (b) classificação dos quadros clínicos; e (c) fatores de risco e proteção. A partir das considerações apresentadas, defende-se como urgente e prioritária a realização de intervenções precoces com caráter preventivo, tendo como alvo evitar ou minimizar os problemas futuros em uma população que pode estar em situação de risco.

Palavras-chave: Saúde mental, psicopatologia, infância, adolescência.

\section{Clinical Manifestation, Classification Models and Risk/Protective Factors for Psychopathology in Childhood and Adolescence}

\begin{abstract}
Although attention to children and adolescents mental health has increased since the beginning of the XXI century, works in this area are still incipient and there is little published in the literature on juvenile mental health intervention in comparison with what has been accomplished with adults. Issues related to children and adolescent psychopathologies have singularities and deserve different considerations regarding the evolution, classification and treatment, especially considering individual development stages. This paper aims to address three aspects associated with children and adolescent mental health that imposes great impact both on the diagnosis and prognosis of emotional and behavioral difficulties, namely: (a) heterogeneous clinical manifestations, (b) clinical classification, and (c) risk and protective factors. From the presented considerations, we defend that early intervention with preventive aspects, aiming to prevent or minimize future problems, targeting the population that may or at risk, are urgent and should be dealt as priority.
\end{abstract}

Keyword: Mental health, psychopathology, infancy, adolescence.

Endereço para correspondência: Rua do Símbolo, 131, apto 72, Jd. Ampliação, São Paulo, SP, Brasil 05713570. E-mail: fabianagauy@gmail.com e marinamonzani@gmail.com 


\section{Manifestación Clínica, Modelos de Clasificación y Factores de Riesgo/Protección de la Psicopatología en la Infancia y Adolescencia}

\section{Resumen}

Aunque la atención de la salud mental de niños y adolescentes ha aumentado desde principios de siglo XXI, el trabajo en esta área es aún incipiente y hay poca literatura publicada sobre intervención en la salud mental de menor en comparación con el trabajo realizado con el público adulto. Las cuestiones relacionadas con la psicopatología juvenil tienen singularidades y merecen consideraciones diferentes sobre la evolución, clasificación y tratamiento, siempre teniendo en cuenta la etapa de desarrollo individual. Este artículo tiene como objetivo hacer frente a tres aspectos relacionados con la salud mental juvenil y de gran impacto tanto en el diagnóstico y pronóstico de las dificultades emocionales y de comportamiento, a saber: (a) las manifestaciones clínicas heterogéneas, (b) la clasificación clínicas de los problemas, y (c) los factores de riesgo y protectores. A partir de las consideraciones presentadas, defendiéndose como urgentes y prioritarias las intervenciones tempranas con aspectos preventivos, dirigida a prevenir o minimizar los problemas futuros en una población que pueden estar en riesgo.

Palabras clave: Salud mental, psicopatología, niños, adolescentes.

Estudos epidemiológicos nacionais e internacionais apontam taxas variadas de prevalências para as psicopatologias na infância e adolescência. No geral, estima-se que de $10 \%$ a $25 \%$ das crianças e adolescentes, em um dado momento, apresentem algum comprometimento considerado clínico ou desviante, necessitando, assim, de tratamento especializado (Fleitlich \& Goodman, 2000; Fleitlich-Bilyk \& Goodman, 2004; Merikangas et al., 2010; Paula, Duarte, \& Bordin, 2007). Adicionalmente às mencionadas taxas de prevalência, cita-se que estudos retrospectivos e prospectivos têm demonstrado que os transtornos que começam na infância e adolescência são preditores de problemas na vida adulta, o que aumenta ainda mais a urgência de intervenções de prevenção e promoção em saúde mental infanto-juvenil (Kessler et al., 2005; McConaughy \& Wadsworth, 2000; Patel, Flisher, Hetrick, \& McGorry, 2007; Patel, Flisher, Nokapota, \& Malhotra, 2008; Paula et al., 2007).

Apesar desses dados, observa-se uma inclusão tardia da saúde mental infanto-juvenil na agenda das políticas públicas, nacional e internacional. Segundo Couto, Duarte e Delgado (2008), isso se deve a pelo menos quatro fatores associados: (a) a variedade dos quadros clínicos - aqui são incluídas a quantidade de transtornos, a comorbidade entre eles e a variação sintomatológica devido ao período do desenvolvimento e ao ambiente em que a criança/adolescente está inserida -, o que desafia os profissionais em relação à avaliação e ao tratamento da referida clientela; (b) os conhecimentos ainda recentes da área, obtidos por estudos epidemiológicos consistentes datados apenas a partir de 1980; (c) a inexistência, até recentemente, de dados sobre tratamentos empiricamente validados; e (d) a dificuldade de inclusão da saúde mental infanto-juvenil na saúde pública.

$\mathrm{Na}$ tentativa de atender essa demanda e minimizar o impacto dos problemas de saúde mental nessa população no Brasil, o Ministério da Saúde implantou 122 Centros de Atenção Psicossocial para a Infância e Adolescência (CAPSi; Couto et al., 2008; Ministério da Saúde, 2010; Santos, 2006). Ainda que isso seja um avanço, este formato contempla apenas o atendimento de transtornos graves. Tal medida é um contrassenso, uma vez os menores de 18 anos representam $40 \%$ da população brasileira e que a maior parte da demanda infanto-juvenil com problemas emocionais e comportamentais não tem o perfil para esse tipo de serviço (Delfini, Dombi-Barbosa, Fonseca, Tavares, \& Reis, 2009; Paula et al., 2007). 
Com o objetivo de contribuir para compreensão da saúde mental infanto-juvenil, este artigo tem como objetivo abordar, utilizando-se de uma revisão bibliográfica não sistemática, três aspectos associados ao tema, com grande impacto tanto no diagnóstico como no prognóstico de dificuldades emocionais e comportamentais, a saber: (a) heterogeneidade das manifestações clínicas; (b) classificação dos quadros clínicos; e (c) fatores de proteção e fatores de risco.

\section{Heterogeneidade das Manifestações Clínicas}

Diferentes estudos evidenciam que há uma heterogeneidade na manifestação clínica das dificuldades da população infanto-juvenil. Wright e Zacriski (2001), por exemplo, solicitaram a 34 professores de escolas especiais que descrevessem os comportamentos de 208 alunos com idade entre seis e 18 anos. Os resultados indicaram que os comportamentos apresentados por crianças com diagnóstico de ansiedade generalizada e depressão foram descritos como isolamento, letargia e anedonia, para algumas, e como agitação e agressividade, para outras. No mesmo estudo foi observado que a situação social envolvida também pode modificar a resposta. Por exemplo, uma criança pode apresentar comportamento agressivo em resposta a eventos considerados aversivos, como ameaça e punição, enquanto outra pode apresentar a mesma topografia comportamental em resposta a eventos considerados positivos, como o elogio de um adulto, ou seja, manifestações comportamentais bastante distintas podem ser encontradas em um mesmo diagnóstico clínico.

Outro fator que favorece a variabilidade na manifestação clínica do transtorno e dificulta sua compreensão é a comorbidade, isto é, a alta prevalência de sobreposição e combinação de diferentes respostas comportamentais e emocionais desadaptativas, como, por exemplo, conjugação de ansiedade e agressividade, depressão e problemas de conduta, diferentes tipos de problemas de saúde mental e queixas escolares, ou mesmo a combinação dessas respostas com condições médicas, como depressão e dor crônica, ou ansiedade e dores de cabeça e no abdômen (Combs-Orme, Heflinger, \& Simpkins, 2002; D’Abreu \& Marturano, 2011; Liu, 2004; Masi, Favilla, Mucci, \& Millepiedi, 2000).

As comorbidades mais frequentes são aquelas entre diferentes problemas comportamentais e emocionais. Angold, Costello e Erkanli (1999), por exemplo, investigaram causas e efeitos de comorbidades entre depressão, transtorno de ansiedade, transtorno de déficit de atenção e hiperatividade (TDAH), transtorno de conduta e transtorno opositor desafiante, considerados como as psicopatologias mais comuns na infância e na adolescência. Esse estudo apontou para uma alta prevalência de comorbidades, especialmente nos casos de TDHA, transtorno de conduta e transtorno opositor desafiante. Kaplan, Dewey, Crawford, e Wilson (2001), por sua vez, avaliaram a sobreposição de dificuldades de desenvolvimento em 179 crianças em idade escolar e encontraram uma prevalência de dificuldades múltiplas quando ocorriam transtorno de leitura, TDAH, transtorno de desenvolvimento da coordenação, transtorno opositor desafiante, transtorno de conduta, depressão e ansiedade. A maior taxa de sobreposição foi nos transtornos de leitura e de déficit de atenção e hiperatividade $(80,4 \%)$. Os autores questionam se essas combinações de sintomas devem ser consideradas comorbidades ou se devem ser compreendidas como manifestações diferentes de uma mesma patologia, tamanha a incidência de sobreposição de quadros clínicos.

Alguns estudos ainda sugerem que dificuldades físicas podem predizer ou favorecer dificuldades emocionais futuras, e vice-versa. Essa relação é mais significante entre os transtornos emocionais, como depressão e ansiedade, e as doenças físicas que afetam a imunidade, como alergias e dificuldades respiratórias. Evidências dessa relação foram encontradas por Combs-Orme et al. (2002), por exemplo. Os autores compararam 965 crianças com doenças clínicas, com ou sem psicopatologias, e mostraram que crianças com problemas emocionais sérios eram significativamente mais suscetíveis a condições clínicas crônicas. Em função disso, sugerem que a integração dos serviços pediátricos e de saú- 
de mental possibilitaria um grande benefício à criança e seus familiares, porque essa junção permitiria um melhor prognóstico do curso das condições físicas facilitadoras das dificuldades emocionais e comportamentais, o que, por sua vez, possibilitaria um melhor planejamento de prevenção e de tratamento.

Masi et al. (2000) citam que também é comum a queixa de somatização, definida como queixas físicas que não podem ser atribuídas a uma patologia clínica, sendo dor de cabeça, falta de energia, dores musculares e abdominais as mais comuns. A partir dessas considerações, os referidos autores investigaram a prevalência de sintomas somáticos em 162 crianças e adolescentes de um serviço de saúde mental, e encontraram queixas somáticas em $69,2 \%$ da amostra, sendo a dor de cabeça a mais frequente $(50,6 \%)$. Foi observado também que crianças mais jovens queixavam-se mais de dores abdominais do que os adolescentes e que os participantes diagnosticados com problemas emocionais, como ansiedade, queixavam-se mais do que os diagnosticados com problemas comportamentais, como agressividade.

Outro fator de variabilidade é o gênero. Os problemas internalizantes, como ansiedade e depressão, são mais comuns entre meninas adolescentes e os problemas externalizantes, como violação de regras e déficit de atenção/hiperatividade, são mais comuns nos meninos na primeira e na média infância (Angold et al., 1999; Rutter, Caspi, \& Moffitt, 2003; Vitolo, Fleitlich-Bilyk, Goodman, \& Bordin, 2005; Zahn-Waxler, Shirtcliff, \& Marceau, 2008). Estudos epidemiológicos têm sugerido ainda que os homens, mais do que as mulheres, abusam de substâncias lícitas ou ilícitas mais pesadas e com maior frequência (Gil, Wagner, \& Tubman, 2004) e que as mulheres apresentam maiores índices de depressão (Seedat et al., 2009).

Uma criança ou um adolescente também pode apresentar uma resposta diferente dos demais devido a uma variação do desenvolvimento normal, ou a uma resposta adaptativa ao ambiente, ou, ainda, a um transtorno transitório, sem caracterizar quadros clínicos (Drotar, 2002). Por exemplo, uma criança pode ter controle esfincte- riano aos dois anos, enquanto outra alcança essa habilidade apenas aos quatro anos, o que ainda é condizente com a idade e o nível do desenvolvimento nesse período; comportamentos agressivos podem ser desenvolvidos como resposta adaptativa a um ambiente hostil e violento; e, ainda, aos dois ou três anos é comum a criança apresentar uma gagueira transitória devido ao aumento do vocabulário.

Os chamados quadros clínicos são respostas desadaptativas ou desviantes, de traços exagerados do desenvolvimento normal que resultam em um padrão atípico, sendo que a disfuncionalidade pode estar no excesso ou na ausência do comportamento. Há ainda controvérsia sobre que parâmetros seriam mais apropriados para o diagnóstico e o prognóstico desses quadros clínicos, e quais variáveis do organismo e do ambiente estariam associadas e deveriam ser consideradas no planejamento das ações de intervenção. Há pelo menos duas considerações a serem feitas: como classificar os problemas e proporcionar uma linguagem comum a diferentes profissionais; e como intervir nas variáveis envolvidas, mantenedoras ou desencadeadoras do problema.

\section{Classificação Diagnóstica dos Quadros Clínicos}

As duas abordagens taxonômicas mais utilizadas em pesquisas com crianças e adolescentes são: Avaliação Baseada em Diagnóstico, derivada de um sistema diagnóstico categórico de sintomas, e Avaliação Empiricamente Baseada, derivada de medidas empíricas dimensionais de síndromes (Achenbach \& Rescorla, 2007). Ambas podem ser utilizadas como bons paradigmas conceituais para ampliar o conhecimento que temos sobre as psicopatologias na infância e na adolescência.

Os sistemas categóricos são descritos no Manual Diagnóstico e Estatístico de Transtornos Mentais - DSM, atualmente na $5^{\text {a }}$ edição - DSM-5, e na Classificação de Transtornos Mentais e de Comportamento da Classificação Internacional de Doenças e de Problemas Relacionados à Saúde, na $10^{\mathrm{a}}$ edição - CID-10, 
e indicam o diagnóstico a partir do reconhecimento, por profissionais experts, de sintomas que ocorrem juntos (American Psychiatric Association [APA], 2013; Organização Mundial de Saúde [OMS], 1993). Por sua vez, o sistema dimensional de síndromes, proposto por Achenbach e Edelbrock em 1978, indica o diagnóstico a partir de escalas empíricas compatíveis, que descrevem as dificuldades infantis em dimensões diferenciadas por síndromes externalizantes e internalizantes. A síndrome externalizante é definida por padrões de comportamentos manifestos desajustados, como agressividade, dificuldades de concentração e comportamento delinquente, enquanto a síndrome internalizante é definida por padrões de comportamentos privados desajustados, como tristeza e isolamento (Achenbach \& Edelbrock, 1978; Achenbach \& Rescorla, 2007; Gerhardt, Compas, Connor, \& Achenbach, 1999).

Os sistemas de categorias diagnósticas são muito difundidos nos serviços de saúde mental e apresentam a vantagem de usar critérios e termos familiares a diferentes especialistas, o que facilita o estabelecimento de uma comunicação homogênea. A novidade desse tema é a mencionada versão do DSM-5, publicada em maio de 2013, agora nomeado em algarismo arábico e não mais algarismo romano. Ao invés de separar os transtornos da infância, nessa nova versão, as psicopatologias são apresentadas com ênfase nas diferentes formas que elas podem assumir no curso da vida. Apesar de tentar contemplar, mais do que as versões anteriores, as questões desenvolvimentais e as variações pessoais (Kraemer, Kupfer, Narrow, Clarke, \& Regier, 2010), a versão atual foi recebida com muitas críticas (Hebebrand \& Buitelaar, 2011; Kraemer et al., 2010; Regier et al., 2013).

A questão central das críticas realizadas foi a de que a ampliação dos critérios diagnósticos poderia gerar um aumento nas taxas de prevalência em diferentes quadros clínicos (Hebebrand \& Buitelaar, 2011; Wing, Gould, \& Gillberg, 2011) e uma possível maior influência de fatores ambientais/culturais, bem como da experiência clínica do pesquisador, no diagnóstico. Além disso, são citadas possíveis implicações comerciais
- favoráveis - dessa ampliação para a indústria farmacêutica (Hebebrand \& Buitelaar, 2011; Regier et al., 2013).

As principais alterações realizadas em comparação à versão anterior do DSM, no que se refere à população infanto-juvenil, foram: (a) exclusão da sessão/capítulo "Transtornos diagnosticados inicialmente na infância e na infância/ adolescência"; (b) proposta de um novo quadro clínico - Transtorno de Desregulação do Humor (Temper Dysregulation Disorder); (c) estabelecimento de mudanças nos critérios diagnósticos para Transtornos Alimentares e TDAH; e (d) proposta de mudança nos critérios diagnósticos para os Transtornos Invasivos do Desenvolvimento, que passou a ser denominado Transtorno do Espectro do Autismo (TEA).

O novo quadro clínico proposto, o Transtorno de Desregulação do Humor, coloca a irritabilidade patológica como diagnóstico distinto e não mais como critério diagnóstico passível de ser incluído nos problemas internalizantes (transtornos de humor - predominantemente bipolar, depressão ou distimia - e transtorno de ansiedade generalizada) e nos problemas externalizantes (transtorno opositivo desafiador). Esse novo diagnóstico vem contrapor o diagnóstico de transtorno bipolar na infância (Regier et al., 2013; Stringaris, 2011).

Nos quadros que compõem os Transtornos Alimentares, a maior mudança foi a troca de Transtorno de Compulsão Periódica para Transtorno de Compulsão Alimentar; a revisão dos critérios para bulimia e anorexia, de maneira que reflitam mais a realidade observada na clínica; e a inclusão de pica e ruminação (Knoll, Bulik, \& Hebebrand, 2010). O diagnóstico de TDAH foi incluído nos Transtornos do Neurodesenvolvimento. Além disso, o critério de início dos sintomas para esse diagnóstico foi alterado de antes de sete anos para antes de 12 anos e passou-se a permitir a comorbidade com transtornos do espectro autista. Outra modificação refere-se à melhor distinção entre os transtornos de conduta e o transtorno opositivo-desafiador (Tannock, 2013).

Por fim, os Transtornos do Espectro do Autismo passam agora a englobar o Autismo, 
Asperger, Transtorno Desintegrativo da Infância e Transtornos Invasivos do Desenvolvimento Sem outras Especificações. Essa junção parte do princípio de que esses quadros apresentam em comum prejuízos no que concerne comunicação, interação social, fixação de interesses e comportamentos repetitivos, com início na infância (Gibbs, Aldridge, Chandler, Witzlsperger, \& Smith, 2012; Regier et al., 2013; Wing et al., 2011). Wing et al. (2011) criticam o fato de os critérios estabelecidos não mencionarem um problema comum a esses pacientes, que é a incapacidade de prever as consequências de suas ações para si mesmo ou para os outros.

Contrariando as críticas apresentadas para o DSM-5, Regier et al. (2013), em um estudo que avaliou a confiabilidade das categorias diagnósticas da nova versão do manual para adultos e crianças/adolescentes nos Estados Unidos e Canadá, observaram que, na população infanto-juvenil estudada, as mudanças testadas para TDAH apresentaram uma confiabilidade muito boa e a mudança para Transtornos do Espectro Autista, confiabilidade boa. Além disso, Polanczyk et al. (2010), em um estudo com o objetivo específico de avaliar o impacto da ampliação da faixa etária para o início dos sintomas (de antes dos sete para antes dos 12 anos) como critério para o diagnóstico de TDAH, encontraram que não deve ocorrer mudança significativa na prevalencência do transtornos. Ainda assim, novos estudos serão necessários para confimar esses dados em outras populações e para as demais categorias diagnósticas.

Apesar dos diferentes modelos de classificação, é fato que ainda não há um entendimento completo sobre a etiologia das psicopatologias; entretanto já existem evidências concretas para afirmar que variáveis ambientais e individuais interagem de maneira complexa para causar os problemas. Por conseguinte, a elaboração de um diagnóstico deve considerar que alguns fatores podem expor (fatores de risco) ou proteger (fatores de proteção) a criança e o adolescente das adversidades ou do desenvolvimento de problemas emocionais e comportamentais. Esse aspecto será abordado a seguir.

\section{Fatores de Proteção e Fatores de Risco}

São conhecidos como fatores de proteção as variáveis que reduzem o efeito do risco, ou seja, que favorecem o crescimento e desenvolvimento saudáveis, ainda que haja exposição da criança ou adolescente à diferentes possibilidades de resultados negativos para a saúde, o bem-estar e o desempenho social (Grant et al., 2006; Sapienza \& Pedromônico, 2005). Autores como Maia e Williams (2005) apontam os três tipos principais de fatores de proteção para o desenvolvimento da criança ou adolescentes: (a) atributos individuais (proximais); (b) atributos familiares (meio-proximais); (c) fontes de apoio individuais ou institucionais (distais). Os fatores proximais ou pessoais são relativos às características do organismo, tais como gênero, temperamento, personalidade e habilidades cognitivas. Por sua vez, os fatores meio-proximais ou familiares são relativos aos ambientes emocional e social da família nuclear, tais como estilo parental e práticas educativas, capacidade de afeição e vínculo, eventos familiares estressantes e presença de psicopatologia em algum ou ambos os pais. Os fatores distais ou sociais são relativos às condições ambientais básicas, como região de moradia, nutrição, violência urbana, acesso a cuidados básicos de saúde e saneamento básico.

Fatores de risco geralmente são compreendidos como a presença de uma característica, ação, evento, ou experiência que aumenta a probabilidade da ocorrência de um problema, ou de consequências adversas para o desenvolvimento ou funcionamento psicológico/social (Assis, Avanci, \& Oliveira, 2009; Gomide, Salvo, Pinheiro, \& Sabag, 2005; Grant et al., 2006; Kraemer et al., 2010). Sabe-se que alguns desses fatores estão relacionados à características individuais, enquanto outros ao seu meio microssocial e outros, ainda, a condições estruturais e socioculturais mais amplas (Zweig, Phillips, \& Lindberg, 2002).

A relação dos estressores ambientais no desenvolvimento e manutenção de psicopatologia na infância e adolescência é discutida no artigo de revisão de Grant et al. (2006). Neste artigo, os 
referidos autores diferenciam os efeitos moderadores e mediadores dos fatores de risco, sendo que as pesquisam que focam nos moderadores se preocupam em identificar que variáveis estressoras estão associadas ao aumento ou redução de quadros psicopatológicos infanto-juvenis, enquanto que os estudos que avaliam os mediadores identificam as condições estressoras necessárias ou preditoras da ocorrência problemas emocionais e comportamentais.

Os dados obtidos por estudos das variáveis moderadoras dos problemas comportamentais e emocionais na infância e adolescência ainda são limitados e incipientes, com exceção dos estudos que apontam uma variável pessoal, as cognições depressiogênicas, como moderadora na relação entre estressores e sintomas depressivos. Por outro lado, os estudos sobre fatores mediadores indicam diversas variáveis que predizem problemas, como, por exemplo, as variáveis familiares associadas como mediadoras para sintomas psicológicos: disciplina inconsistente, relacionamento/vínculo pobre entre pais e filhos, negligência, baixo envolvimento materno na escola, falta de rotina familiar e conflito familiar. Ainda dentre as variáveis familiares, a questão do divórcio e conflito conjugal também são apontadas como mediadoras de dois tipos: (a) baseados na família - tais como isolamento/rejeição, descontrole emocional, hostilidade e mudanças negativas na relação pai e filho; e (b) baseados na criança, como dificuldade de enfrentamento e baixo controle externo (Grant et al., 2006).

Alguns estudos brasileiros com foco na família têm sugerido que a pobreza, a residência em zona rural e a baixa escolaridade estão associados a menor conhecimento sobre o curso normal do desenvolvimento, e que estas variáveis podem mediar a relação estresse e psicopatologia na infância e adolescência (Assis et al., 2009; Gauy \& Guimarães, 2006; Moura et al., 2004). Sabe-se também que pais ou cuidadores com doença mental, baixas habilidades sociais e estressados avaliam os filhos com mais problemas e utilizam práticas educativas predominantemente negativas, como humor inconsistente, negligência e punição física (Gomide et al., 2005). Tal afirmação é corroborada pelo estudo de Salvo,
Silvares e Toni (2005), que indica a falta de monitoria positiva, aliada à presença de práticas educativas negativas, como variáveis preditoras de problemas de comportamento.

\section{Considerações Finais}

Diante do exposto, fica evidente que os quadros psicopatológicos geram custos diretos e indiretos de grande impacto, tanto na vida pessoal e familiar quanto nas instituições acadêmicas e de saúde. Estima-se que esse impacto ainda é maior nos países subdesenvolvidos e em desenvolvimento, aqui inclui-se o Brasil, devido ao fato de que nesses países houve uma rápida urbanização das cidades, fator de risco associado a aumento da pobreza e desemprego (Assis et al., 2009; Patel et al., 2008); e de que nesses países há uma grande população infanto-juvenil, que consiste em $35-50 \%$ da população (Patel et al., 2008).

O reduzido nível de atenção à saúde mental, de forma ampla, e à população infanto-juvenil, em particular, configura, assim, um problema grave e não restrito ao Brasil. Apesar da elevada demanda por atendimento nessa área, na maioria dos países apenas $1 \%$ dos orçamentos destinados à saúde são dirigidos especificamente à saúde mental (OMS, 2002). Além disso, estima-se que mais de $30 \%$ dos países não têm programas na área e mais de $90 \%$ não têm políticas de saúde mental que incluam crianças e adolescentes (Belfer, 2008; Couto et al., 2008; OMS, 2002; Santos, 2006).

Dessa forma, considerando a prevalência dos problemas de saúde mental na população infanto-juvenil e seus relevantes impactos sobre o desenvolvimento humano e social, constata-se a necessidade premente de que profissionais clínicos e pesquisadores da área dediquem mais esforços para a produção de conhecimento e de soluções para a prevenção e o atendimento a essa faixa etária. A literatura evidencia que o desenvolvimento dessa área da saúde deve contemplar uma multiplicidade de aspectos, entre os quais se destacam os fatores mencionados neste trabalho de revisão: a heterogeneidade das manifestações clínicas, que representa um grande desafio tan- 
to para o atendimento a crianças e adolescentes, quanto para o desenvolvimento de métodos e protocolos que possam propiciar maior eficiência e efetividade aos tratamentos; a classificação diagnóstica dos quadros clínicos, que abarca a definição de padrões para diagnósticos mais acurados e seguros, capazes de orientar os profissionais clínicos e criar condições favoráveis à construção de mais conhecimento sobre a área; e uma compreensão mais profunda sobre os fatores de risco e proteção, necessária para o delineamento de estratégias preventivas e para um melhor entendimento dos quadros clínicos, em função do seu contexto.

A discussão sobre os referidos fatores abordados neste trabalho, além de evidenciar a complexidade do tema e caracterizar os desafios para os profissionais clínicos e pesquisadores, também traz indícios relevantes sobre aspectos e diretrizes que devem pautar a formulação e a implementação de políticas públicas direcionadas aos cuidados em saúde mental da população infanto-juvenil. Nesse sentido, sugere-se que a qualificação profissional especializada deva ser considerada como uma alternativa necessária para a atuação na prevenção e no tratamento de problemas de saúde mental de crianças e adolescentes. Ao mesmo tempo, indica-se que a ponderação mais ampla sobre os fatores de risco e proteção pode orientar a criação de programas de saúde pública que contemplem mecanismos de atuação multidisciplinar, oferecendo maior qualidade para o atendimento dos pacientes, de forma concomitante à elaboração de modelos preventivos com maior efetividade.

Ademais, o aprimoramento necessário da atuação governamental vai ao encontro da priorização do atendimento regionalizado, que constitui um dos eixos do modelo de atendimento em saúde mental em consolidação no Brasil (Delfini et al., 2009), permitindo um atendimento mais próximo à população-alvo e uma melhor compreensão dos contextos em que emergem as dificuldades de desenvolvimento humano e social. Todavia, em que pese o valor intrínseco a esse modelo de regionalização, ainda se observa um despreparo do setor público para atender a demanda infanto-juvenil, o que é evidenciado pela ausência de profissionais de saúde mental nas equipes do Programa da Saúde da Família em muitas regiões brasileiras, e pela ainda reduzida sensibilidade para questões emocionais por parte dos profissionais das unidades básicas de saúde (Tanaka \& Ribeiro, 2009). Além disso, prevalece uma flagrante desarticulação entre diferentes setores públicos, como justiça, saúde e educação (Couto et al., 2008), o que contribui para reduzir a eficiência no uso dos recursos e limitar a consecução de resultados para crianças e adolescentes, suas famílias e a coletividade.

Somam-se a esses aspectos as dificuldades relativas à elevada demanda por atendimento psicológico, demonstrada em dados de levantamento sobre os serviços de psicologia e serviços-escola: em regra, esses serviços têm como características as longas filas de espera, resultando em desistências frequentes; o acesso difícil para os usuários, tanto pela localidade quanto pela indisponibilidade de transporte público; e o alto custo, uma vez que o formato tradicional de atendimento individual, com frequência semanal e de longa duração, ainda é mantido como forma preferencial (Gauy \& Guimarães, 2006; Prebianchi \& Cury, 2005).

Conclui-se, portanto, que a superação desse cenário atual requer uma atuação conjunta de profissionais clínicos, da academia e do poder público. A coordenação desses atores pode contribuir para o enfrentamento dos fatores que tornam a saúde mental infanto-juvenil um tema de elevada complexidade clínica e um desafio coletivo sob a perspectiva da saúde pública. Como mencionado na introdução a este trabalho, as questões relativas à psicopatologia infanto-juvenil possuem especificidades que requerem considerações próprias quanto a evolução, classificação e tratamento dos problemas, considerados sempre em relação ao contexto e aos estágios do desenvolvimento individual. Por conseguinte, emerge como urgente e prioritária a geração e a disseminação de mais conhecimento sobre o tema e a criação de condições para a intervenção precoce, especialmente de caráter preventivo, visando à minimização dos transtornos que atingem crianças e adolescentes, trazendo ganhos para sua saúde no presente e reduzindo os problemas decorrentes que poderiam enfrentar no futuro. 


\section{Referências}

Achenbach, T. M., \& Edelbrock, C. S. (1978). The classification of child psychopathology: A review and analysis of empirical efforts. Psychology Bulletin, 85(6), 1275-1301. doi:10.1037/00332909.85.6.1275

Achenbach, T. M., \& Rescorla, L. A. (2007). Multicultural understanding of child and adolescent psychopathology. New York: Guilford Press.

American Psychiatric Association. (2013). Diagnostic and statistical manual of mental disorders ( $5^{\text {th }}$ ed.). Arlington, VA: Author.

Angold, A., Costello, E. J., \& Erkanli, A. (1999). Comorbidity. The Journal of Child Psychology and Psychiatry and Allied Disciplines, 40, 57-87.

Assis, S., Avanci, J. Q., \& Oliveira, R. V. C. (2009). Desigualdades socioeconômicas e saúde mental infantil. Revista de Saúde Pública, 43(1), 92 100.

Belfer, M. L. (2008). Child and adolescent mental disorders: The magnitude of the problem across the globe. The Journal of Child Psychology and Psychiatry, 49(3), 226-236. doi:10.1111/j.14697610.2007.01855.x

Combs-Orme, T., Heflinger, C. A., \& Simpkins, C. G. (2002). Comorbidity of mental health problems and chronic health conditions in children. Journal of Emotional and Behavioral Disorders, 10(2), 116-125. doi:10.1177/106342660201000 20601

Couto, M. C. V., Duarte, C. S., \& Delgado, P. G. G. (2008). A saúde mental infantil na saúde pública brasileira: Situação atual e desafios. Revista Brasileira de Psiquiatria, 30(4), 390-398.

D’Abreu, L. C. F., \& Marturano, E. M. (2011). Identificação de problemas de saúde mental associados à queixa escolar segundo a DAWBA. Psico, $42,152-158$.

Delfini, P. S. S., Dombi-Barbosa, C., Fonseca, F. L., Tavares, C. M., \& Reis, A. O. A. (2009). Perfil dos usuários de um centro de atenção psicossocial infantojuvenil da grande São Paulo, Brasil. Revista Brasileira do Crescimento e Desenvolvimento Humano, 19(2), 226-236.

Drotar, D. (2002). Enhancing reviews of psychological treatments with pediatric populations: Thoughts on next steps. Journal of Pediatric Psychology, 27(2), 167-176.
Fleitlich, B. W., \& Goodman, R. (2000). Epidemiologia. Revista Brasileira de Psiquiatria, 22(Supl. 2), 2-6.

Fleitlich-Bilyk, B., \& Goodman, R. (2004). Prevalence of child and adolescent psychiatric disorders in Southeast Brazil. Journal of the American Academy of Child and Adolescent Psychiatry, 43, 727-734. doi:10.1097/01. chi.0000120021.14101.ca

Gauy, F. V., \& Guimarães, S. S. (2006). Triagem em saúde mental infantil. Psicologia: Teoria e Pesquisa, 22, 5-16.

Gerhardt, C. A., Compas, B. E., Connor, J. K., \& Achenbach, T. M. (1999). Association of mixed anxiety-depression syndrome and symptoms of major depressive disorder during adolescence. Journal of Youth and Adolescence, 28(3), 305-323.

Gibbs, V., Aldridge, F., Chandler, F., Witzlsperger, E., \& Smith, K. (2012). Brief report: An exploratory study comparing diagnostic outcomes for autism spectrum disorders under DSM-IV-TR with the proposed DSM-5 revision. Journal of Autism and Development Disorders, 42, 17501756. doi:10.1007/s10803-012-1560-6

Gil, A. G., Wagner, E. F., \& Tubman, J. G. (2004). Culturally sensitive substance abuse intervention for Hispanic and African American adolescents: Empirical examples from the Alcohol Treatment Targeting Adolescents in Need (ATTAIN) Project. Addiction, 99(2), 140-150. doi:10.1111/j.1360-0443.2004.00861.x

Gomide, P. I. C., Salvo, C. G., Pinheiro, D. P. N., \& Sabag, G. (2005). Correlação entre práticas educativas, depressão, estresse e habilidades sociais. Psico-USF, 10(2), 169-178.

Grant, K. E., Compas, B. E., Thurm, A. E., McMahon, S., Gipson, P. Y., Campbell, A. J., ... Westerholm, R. I. (2006). Stressors and child and adolescent psychopathology: Evidence of moderating and mediating effects. Clinical Psychology Review, 26, 257-283. doi:10.1016/j. cpr.2005.06.011

Hebebrand, J., \& Buitelaar, J. K. (2011). On the way to DSM-V. European Child \& Adolescent Psychiatry, 20, 57-60. doi:10.1007/s00787-010-0157-x

Kaplan, B. J., Dewey, D. M., Crawford, S. G., \& Wilson, B. N. (2001). The term comorbidity is of questionable value in reference to developmental disorders. Journal of Learning Disabilities, 34(6), 555-565. doi:10.1177/002221940103400608 
Kessler, R. C., Berglund, P., Demler, O., Jin, R., Merikangas, K. R., \& Walters, E. E. (2005). Life-time prevalence and age-of-onset distribution of DSM-IV disorders in the National Comorbidity Survey Replications. Archive of General Psychiatry, 65, 593-602. doi:10.1001/ archpsyc.62.6.593

Knoll, S., Bulik, C. M., \& Hebebrand, J. (2010). Do the currently proposed DSM-5 criteria for anorexia nervosa adequately consider development aspects in children and adolescents? European Child \& Adolescent Psychiatry, 20(2), 95-101. doi:10.1007/s00787-010-0141-5

Kraemer, H. C., Kupfer, D. J., Narrow, W. E., Clarke, D., \& Regier, D. A. (2010). Moving toward DSM-5: The field trials. American Journal Psychiatry, 167(1), 1158-1160. doi:10.1176/appi. ajp.2010.10070962

Liu, L. (2004). Childhood externalizing behavior: Theory and implications. Journal of Child and Psychiatric Nursing, 17, 93-103. doi:10.1111/j.1744-6171.2004.tb00003.x

Maia, J. M. D., \& Williams, L. C. A. (2005). Fatores de risco e fatores de proteção ao desenvolvimento infantil: Uma revisão da área. Temas em Psicologia, 13(2), 91-103.

Masi, G., Favilla, L., Mucci, M., \& Millepiedi, S. (2000). Depressive comorbidity in children and adolescents with generalized anxiety disorder. Child Psychiatry and Human Development, 30(3), 205-215. doi:10.1023/A:1021351722364

McConaughy, S. H., \& Wadsworth, M. E. (2000). Life history reports of young adult previously referred for mental health services. Journal of Emotional and Behavioral Disorders, 8, 202215. doi:10.1177/106342660000800401

Merikangas, K. R., He, J.-P., Brody, D., Fisher, P. W., Bourdon, K., \& Koretz, D. S. (2010). Prevalence and treatment of mental disorders among US children in the 2001-2004 NHAES. Pediatrics, 125(1) 75-81. doi:10.1542/peds.2008-2598

Ministério da Saúde. (2010). Relatório final da IV Conferência Nacional de Saúde Mental - Intersetorial. Brasília, DF: Autor.

Moura, M. L. S., Ribas, R. C., Jr., Piccinini, C. A., Bastos, A. C. S., Magalhães, C. M. C., Vieira, M. L., ...Silva, A. K. (2004). Conhecimento sobre desenvolvimento infantil em mães primíparas de diferentes centros urbanos do Brasil. Estudos de
Psicologia (Natal), 9(3), 421-429. doi:10.1590/ S1413-294X2004000300004

Organização Mundial de Saúde. (1993). Classificação de transtornos mentais e de comportamento da CID-10: Descrições clínicas e diretrizes diagnósticas. Porto Alegre, RS: Artes Médicas.

Organização Mundial de Saúde. (2002). XXIV Relatório Mundial da Saúde. Saúde mental: Nova concepção, nova esperança (Escritório Central da Oficina Pan-americana de Saúde, Trad.). Genebra, Suíça: Autor.

Patel, V., Flisher, A., Hetrick, S., \& McGorry,P.(2007). Mental health of young people: A global public-health challenge. The Lancet, 369(9569), 13021313. doi:10.1016/S0140-6736(07)60368-7

Patel, V., Flisher, A. J., Nokapota, A., \& Malhotra, S. (2008). Promoting child and adolescent mental health in low and middle income countries. The Journal of Child Psychology and Psychiatry, 49(3), 313-334.

Paula, C. S., Duarte, S. C., \& Bordin, I. A. S. (2007). Prevalência de problemas de saúde mental em crianças e adolescentes da região metropolitana de São Paulo: Necessidade de tratamento e capacidade de atendimento. Revista Brasileira de Psiquiatria, 29(1), 11-17.

Polanczyk, G., Caspi, A., Houts, R., Kollins, S. H., Rohde, L. A., \& Moffitt, T. E. (2010). Implications of extending the ADHD age-of-onset criterion to age 12: Results from a prospectively studies birth cohort. Journal of the American Academy of Child and Adolescent Psychiatry, 49(3), 210-216. doi:10.1016/j.jaac.2009.12.014

Prebianchi, H. B., \& Cury, V. E. (2005). Atendimento infantil numa clínica-escola de psicologia: Percepção dos profissionais envolvidos. Paidéia (Ribeirão Preto), 15(31), 249-258.

Regier, D. A., Narrow, W. E, Clarke, D. E., Kraemer, H. C., Kuramoto, J., Kuhl, E. A., \& Kupfer, D. J. (2013). DSM-5 Field trials in the United States and Canada, part II: Test-retest reliability of selected categorical diagnoses. American Journal of Psychiatry, 170(1), 59-70. doi:10.1176/appi. ajp.2012.12070999

Rutter, M., Caspi, A., \& Moffitt, T. E. (2003). Using sex difference in psychopathology to study causal mechanisms: Unifying issues and research strategies. Journal of Child Psychologu and Psychiatry, 44(8), 1092-115. doi:10.1111/14697610.00194 
Salvo, C. G., Silvares, E. F. M., \& Toni, P. M. (2005). Práticas educativas como forma de predição de problemas de comportamento e competência social. Estudos de Psicologia (Campinas), 22(2), 187-195. doi:10.1590/ S0103-166X2005000200008

Santos, P. L. (2006). Problemas de saúde mental de crianças e adolescentes atendidos em um serviço público de psicopatologia infantil. Psicologia em Estudo, 11(2), 615-321.

Sapienza, G., \& Pedromônico, M. R. M. (2005). Risco, proteção e resiliência no desenvolvimento da criança e do adolescente. Psicologia em Estudo, 10(2), 209-216.

Seedat, S., Scott, K. M., Angermeyer, M. C., Berglund, P., Bromet, E. J., Brugha, T. S., \& Kessler, T. (2009). Cross-national association between gender and mental disorders in the WHO World Mental Health Surveys. Archives of General Psychiatry, 66(7), 785-795. doi:10.1001/ archgenpsychiatry.2009.36

Stringaris, A. (2011). Irritability in children and adolescents: A challenge for DSM-5. European Child \& Adolescent Psychiatry, 20(2), 61-66. doi:10.1007/s00787-010-0150-4

Tanaka, O. Y., \& Ribeiro, E. L. (2009). Ações de saúde mental na atenção básica: Caminho para ampliação da integralidade da atenção. Ciência \& Saúde Coletiva, 14(2), 477-486.

Tannock, R. (2013). Rethinking ADHD and LD in DSM-5: Proposed changes in diagnostic criteria. Journal of Learning Disabilities, 46(1), 5-25. doi:10.1177/0022219412464341
Vitolo, Y. F., Fleitlich-Bilyk, B., Goodman, R., \& Bordin, I. A. (2005). Parental beliefs and childrearing attitudes and mental health problems among schoolchildren. Revista de Saúde Pública, 39, 716-724.

Wing, L., Gould, J., \& Gillberg, J. (2011). Autism spectrum disorders in the DSM-V: Better or worse than the DSM-IV? Research in Developmental Disabilities, 32, 768-773. doi:10.1016/j. ridd.2010.11.003

Wright, J. C., \& Zacriski, A. L. (2001). A contextual analysis of externalizing and mixed syndromal similarity obscures functional dissimilarity. Journal of Consulting and Clinical Psychology, 69(3), 457-470.

Zahn-Waxler, C., Shirtcliff, E. A., \& Marceau, K. (2008). Disorders of childhood and adolescence: Gender and psychopathology. Annual Review of Clinical Psychology, 4, 275-303. doi:10.1146/ annurev.clinpsy.3.022806.091358

Zweig, J. M., Phillips, B. S., \& Lindberg, L. D. (2002). Predicting adolescent profiles of risk: Looking beyond demographics. Journal of Adolescent Health 31, 343-353. doi:10.1016/S1054139X(02)00371-3 